\title{
IV-Inspektionen, Rentenkürzungen und ärztliche Pflichten
}

\author{
Michel Romanens ${ }^{a}$, Edward A. Schoberb, Werner A. Dislerc, Zoran Kontic ${ }^{d}$, Bernhard Hofmeier \\ Walter Warmuth ${ }^{f}$, Simon Heiniger ${ }^{g}$, Arnold Amacher ${ }^{h}$ \\ ${ }^{a}$ Dr. med., Präsident Verein Ethik und Medizin Schweiz VEMS, PhD; b Dr. med., Hausarzt; ${ }^{c}$ Dipl.-Psych., Eidg. Psychotherapeut SBAP, Psychoanalytiker SGP \\ ${ }^{d}$ Dr., Facharzt für Psychiatrie und Psychotherapie; ${ }^{~}$ Dr. med., Hausarzt und Stiftungsratsmitglied Fairfond; ${ }^{\dagger}$ Dr. rer. nat. habil., Stiftungsratsmitglied Fairfond; \\ ${ }^{g}$ Dr. med., Hausarzt, Olten; ${ }^{h}$ Dr. med., Spezialarzt Pädiatrie und pädiatrische Pneumologie
}

1 http://www.20min.ch/ schweiz/news/story/ IV-sparte-dank-Detektiven-60-Millionenein-10135466

2 https://www.tagesanzeiger.ch/schweiz/standard/ sozialbetrug-zerstoert das-vertrauen/ story/22542742

3 https://www.parlament. ch/de/ratsbetrieb/suchecuria-vista/geschaeft? Affairld=2008110
Laut einer Umfrage von Tamedia ${ }^{1}$ wird die verdeckte Überwachung der IV-Inspektoren von 62\% der Bürgerinnen und Bürger akzeptiert. Ebenso einhellig scheint die Meinung über Ärztinnen und Ärzte, die sich gegen solche Überwachungsmethoden stellen: Sie seien entweder den Versicherungsbetrügern auf den Leim gegangen oder verdienten mit ihnen oder beides. Rudolf Strahm geht in einer Kolumne im Tages-Anzeiger vom 24. April 2018 sogar so weit, den Spiess umzudrehen und zu behaupten: "Wer die Observation und Missbrauchsbekämpfung behindert, unterhöhlt ungewollt unseren Sozialstaat.»² Handeln Ärztinnen und Ärzte also verantwortungslos, wenn sie sich für die Wahrung der Integrität ihrer invaliden Patientinnen und Patien-

\section{Zusammenfassung}

Betrug bei IV-Rentenbezügern und Sozialhilfe-Empfängern ist selten. Die fehlende Transparenz bei der Entscheidung zur Anordnung von Überprüfungen jeglicher Art, insbesondere auch von Observationen, obliegt heute der Versicherung/Gemeinde, welche in diesem Kontext nicht als unabhängige Behörde agiert und damit Partei ist. Dies widerspricht rechtsstaatlichen Grundprinzipien. Die heutige Praxis bei den Überprüfungen sowie die Anpassungen des Gesetzes zu den Observationen (ATSG Art. 43ff.) dienen nicht dazu, Betrüger zu finden, sondern möglichst viele Renten zu kürzen, die eigentlich korrekterweise gewährt wurden. Die hier vorgeschlagene Anpassung der Gesetze, wonach nur eine unabhängige paritätische Kommission eine Überprüfung anordnen darf, dient dazu, den Missbrauch von Überprüfungen zu verhindern und die Gründe für die Anordnungen transparent zu gestalten sowie eine Begleitforschung zur Häufigkeit von tatsächlichem Missbrauch auf nationaler Ebene einzuleiten. Die vorgeschlagene Änderung des Gesetzes führt die Observationen und die Überprüfungspraxis auf eine Inspektionsgrundlage zurück. Damit ist sichergestellt, dass der Verdächtigte die Möglichkeit hat, sich dem Verdacht zu stellen und damit unnötige oder falsch indizierte Observationen und behördliche Massnahmen zu vermeiden. Die Diskussion um korrekte Handhabung bei Verdacht auf Versicherungsbetrug ist umso wichtiger, als aktuell ein Zustandekommen des «Referendums gegen Versicherungsspitzelei» erreicht wurde.

ten einsetzen? Eine rhetorische Frage, es ist klar: Wir sind einzig dem Patientenwohl verpflichtet, denn mit den Patienten stehen wir in einem Auftragsverhältnis. Damit geraten wir unter Umständen aber in einen Konflikt mit den Behörden.

\section{Taugen die Tests der IV aus medizinischer Sicht?}

Wenn die Behörden davon ausgehen, den Anspruch auf Versicherungsleistungen sicher zu erkennen (Sensitivität $100 \%$ ), dann ist dies erstens nicht belegt und unterschlägt zweitens die Population der IV-Berechtigten, die gar keinen Antrag stellen. Aus medizinischer Sicht stellt sich die Frage, was wir von Observationen erwarten können und wer diese auslösen soll. Die bereits seit bald zehn Jahren verwendeten unveröffentlichten Checklisten der IV sollen der Behörde helfen, einen Anfangsverdacht zu erzeugen. Die wissenschaftlich nicht validierte und nicht publizierte Liste umfasst 19 Verdachtsvariablen mit insgesamt 138 möglichen Punkten. Davon genügen 20 Punkte, um ein Verfahren einzuleiten. ${ }^{3}$ Beispiel: Ein Schmerzpatient (5 Punkte) wird vom Nachbar anonym denunziert (20 Punkte). Der IV-Beamte vermutet Malingering (Simulation, Aggravation) aufgrund früherer Gutachten (20 Punkte) und häufigen Arztwechsels (5 Punkte) sowie häufigen Aufenthalts im Ausland (5 Punkte) wegen Migrationshintergrund (3 Punkte). Wie soll Malingering nun aber erkannt werden? Der SFSS-Test (Strukturierter Fragebogen Simulierter Symptome) wird als Beweismittel für Aggravation und Simulation verwendet. Gemäss Literatur ist dieser forensische Test bei Versicherten mit Depressionen allerdings wegen der niedrigen Spezifizität medizinisch falsch gewählt, denn er hat eine Sensitivität von rund 90\% und eine Spezifität von nur 60\%. Das Verhalten entsprechend Malingering gehört zum Krankheitsbild einer Depression oder eines chronischen Schmerzsyndroms. Gemäss Bayes-Theorem ist die diagnostische Genauigkeit 
in Relation zur vom Untersucher erwarteten Wahrscheinlichkeit zu setzen, in diesem Fall für Malingering. Dies bedeutet auch, dass das Ergebnis der Prüfung von der Erwartung des Prüfers abhängt, der Rentenbezüger würde betrügen. Tatsache ist, dass der Test aufgrund der niedrigen Spezifität die Vortestwahrscheinlichkeit für Betrug kaum verändert. Geht der Prüfer von einer Betrugswahrscheinlichkeit von $5 \%$ aus, ergibt ein positiver Test nur eine Wahrscheinlichkeit von $11 \%$, dass dem auch tatsächlich so ist. Folgt schliesslich ein Observationsauftrag, so muss die Validität eines "positiven» Ergebnisses erneut in Frage gestellt werden. Denn eine Observation ist auch hier nur ein Testverfahren ohne bisherige wissenschaftliche Grundlage. Observationen können sinnvoll sein, wenn eine Person Gegenstände entwendet. Dabei ertappt, ist der Beweis erbracht. Bei Patienten mit chronischen Schmerzen oder Depressionen bringen "Beobachtungen" durch nicht-medizinisch geschulte Personen nichts, weil weder Schmerzen noch eine Depression beobachtet werden können.

\section{Taugen die Massnahmen der IV aus ökonomischer Sicht?}

Nicht nur ist die Validität solcher Abklärungen zweifelhaft, sie können auch die Gesamtkosten bei Kranken und Behinderten erhöhen. Einerseits durch die Gutachten selbst: Wird eine medizinische Überprüfung veranlasst, so werden hohe Summen für die Gutachten bezahlt (6000 bis 10000 Franken pro Gutachten). Gleichzeitig müssen mehrere Gutachten übereinstim-

\section{Wird eine medizinische Überprüfung veranlasst, so werden hohe Summen für die Gutachten bezahlt.}

mend die gleiche oder sehr ähnliche Meinungen vertreten, was die Abklärungskosten nochmals deutlich erhöht. Gemäss NZZ vom 23.3.2018 kosten IV-Gutach- ten knapp 100 Mio. Franken pro Jahr: Bei jährlichen Kosten pro IV-Rentner von 15000 Franken entspricht dies 3\% aller IV-Renten. Dazu entstehen noch Observationskosten von 10000 Franken, somit weitere 66 Mio. Franken. Den Ausgaben von 165 Mio. Franken stehen die potentiellen Einnahmen gegenüber. Diese betra-

\section{Medizinisch unqualifizierte Entscheide führen} zu einer Verschlechterung des gesundheitlichen Zustands und damit zu vermeidbaren Behandlungskosten.

gen bei einer Überführungsquote von nur $1 / 3$ und eingesparten Kosten von durchschnittlich 211500 Franken in 10 Jahren pro Vollrentenentzug 518 Mio. Franken. Die Anwaltskosten für die IV-Rentner und immaterielle Schäden sind dabei noch nicht eingerechnet. Gravierender: Medizinisch unqualifizierte Entscheide führen zu einer Verschlechterung des gesundheitlichen Zustands und damit zu vermeidbaren Behandlungskosten. Gesundheitliche Folgen negativer Rentenentscheide trotz Behinderung wurden von der IV bisher aber ungenügend erforscht, wie Frau Dr. med. Doris Brühlmeier in einem wegweisenden Artikel in der SÄZ belegen konnte. ${ }^{4}$

Unsere auf www.kardiolab.ch/rentenlupe.htm durchgeführte Umfrage erfasste in einem anonymisierten Fragebogen Name und Postleitzahl des meldenden Arztes, E-Mail des Arztes, Initialen, Geschlecht und Geburtsdatum der Patienten, die Art der Erkrankung (Schmerz, Psychiatrie, Somatik, Kombination), den Rentenentscheid (noch nicht entschieden, gestoppt, gekürzt, erhöht, neu zugesprochen), den beobachteten Effekt dieses Entscheides auf den weiteren Krankheitsverlauf (unverändert, verbessert, verschlechtert), ob der Patient zwischenzeitlich verstorben ist (dann mit Todesjahr) und die vermuteten Versorgungskosten der Krankheit (weniger, gleich, verdoppelt, verfünffacht, verzehnfacht, weiss nicht). Untenstehende Grafik fasst die Ergebnisse zusammen.
4 https://saez.ch/de/article/ doi/saez.2017.06023/

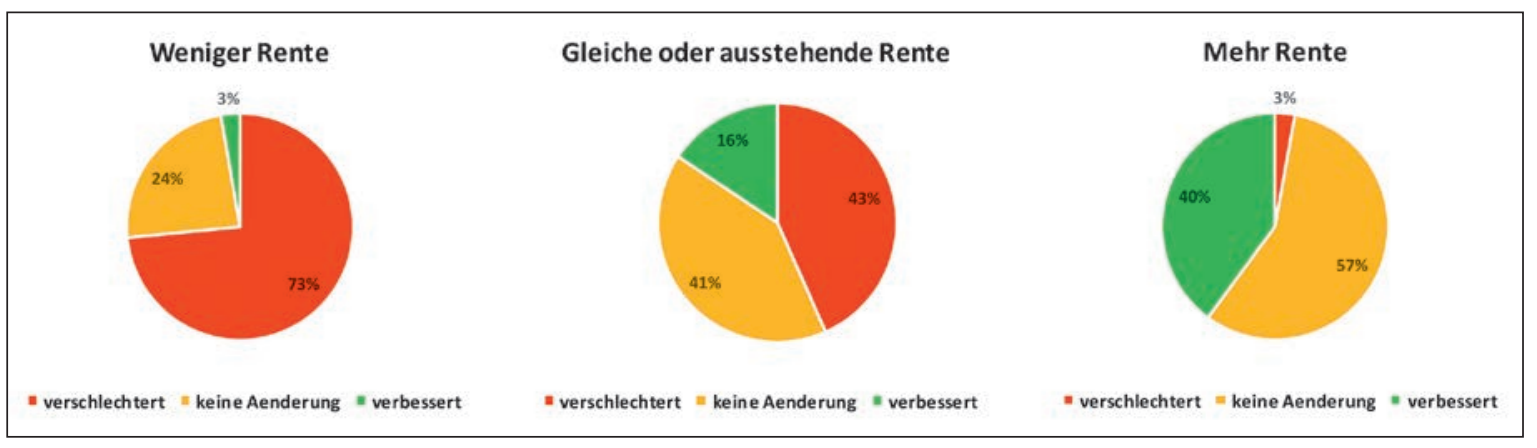

Effekt des Rentenentscheides auf die Gesundheit bei 408 Patienten. 
Die Auswertung zeigt, dass eine Minderung oder die Verweigerung einer Rente bei Patientinnen und Patienten mit Behinderung bei 73\% zu einer Verschlechterung der Gesundheit führte, im umgekehrten Fall nur bei 3\%. Die Aussagekraft dieser Beobachtung kann verbessert werden, wenn die Zahl der Meldungen höher ist (z.B. über 2000). Einschränkung: Da wir lediglich Fälle sammeln, jedoch keine zufällige Stichprobe erhoben haben, besteht die Möglichkeit eines Selektions-Bias. Auch würden die Angaben nicht anhand von Peer-Reviews der Krankengeschichten überprüft.

\section{Sind die Massnahmen pädagogisch sinnvoll?}

Die in der Sozialversicherung angewandten Methoden gehören praktisch insgesamt zur erzieherischen Pädagogik. Es handelt sich um pädagogische Intentionen, die auf Probleme angewendet werden, die auf diesem Wege allerdings nicht zu lösen sind, im Gegenteil: Psychische Probleme, die nur therapeutisch gelöst werden können, werden durch erzieherische Forderungen verstärkt, nicht gelöst. Die von der Justiz eingebrachten Forderungen nach Aufwand des Willens, um psychische Symptome zu «überwinden», ist unsachgemäss. Die Verantwortung, welche die Versicherung zu tragen hat, wird dem Versicherten überantwortet, anstatt dass ihm geholfen wird. Die kranke Person, die am wenigsten in der Lage ist, Verantwortung zu übernehmen, wird durch diese Verantwortungszuschreibung schwer belastet und somit tiefer in ihre Pathologie gedrängt. Diese Problematik ist auch im administrativen Bereich in Interaktionen mit der Invalidenversicherung bereits bei der Anmeldung zu beobachten. Hier geht die IV zurück zu Methoden, die sich etwa in der Suchtprävention und -therapie der achtziger Jahre hinlänglich als kontraproduktiv erwiesen haben. Erst als diese Ansätze als falsch erkannt und überwunden wurden, war der Weg frei für die Heilung der Suchtkranken, während die bis dahin angewandte pädagogische Methode deren Suchtkrankheit verstärkt hat.

\section{Sind die Massnahmen der IV überhaupt rechtskonform?}

Die Patientengutachten der Invalidenversicherung werden heute stärker gewertet als die Einschätzung der behandelnden Ärztinnen und Ärzte. Hier stellen sich verfahrensrechtliche Fragen. ${ }^{5}$ Auch stellt sich die Frage der Unabhängigkeit der von der Invalidenversicherung finanzierten Gutachter, insbesondere da, wo die Invalidenversicherung bei einer Einschätzung, die Rente sei nicht zu streichen, einfach weitere Gutachten einholt, bis sie von einem Gutachter die gewünschte Einschätzung erhält, ${ }^{6}$ oder Gutachter beauftragt, die bekannt sind für versicherungsfreundliche Beurteilung. ${ }^{7}$ Auch stellt sich die Haftungsfrage: Im Bundesgesetz über die Invalidenversicherung (IVG) ist zum Verhältnis UV und MV zu lesen: «Die Prognose über den übertherapeutischen Effekt der Rentenverweigerung muss an sich mit (überwiegender) Wahrscheinlichkeit gestellt werden können.» Das Gesetz geht also davon aus, Rentenverweigerung führe zur beruflichen Integration. ${ }^{8}$ Die Intention der Rentenverweigerung ist somit die einer therapeutischen Massnahme. Damit trifft die Invalidenversicherung einen Behandlungsentscheid, und als solcher muss die Massnahme die Kriterien der WZW-Regel des KVG bezüglich Wirksamkeit, Zweckmässigkeit und Wirtschaftlichkeit erfüllen. Wenn die Versicherer keine entsprechenden Regressforderun-

\section{Das Bundesgesetz zur Invalidenversicherung lässt der Versicherung und ihren Gutachtern einen ungebührlichen Freiraum.}

gen an die IV stellen, so stellt sich die Frage, ob dies eine Verweigerung ihrer in der WZW-Regel des KVG formulierten Pflicht darstellt. Darüber hinaus werden die Ergebnisse der Observationen nicht im Rahmen eines unabhängigen Verfahrens beurteilt, sondern willkürlich bewertet. Ein vages Indiz wird zum Beweis erhoben und weitere Verfahren eingeleitet oder direkt die Rente entzogen. ${ }^{9}$ Bezüglich der Rechtsgrundlagen ist also einiges unklar. Das Bundesgesetz zur Invalidenversicherung lässt der Versicherung und ihren Gutachtern einen ungebührlichen Freiraum. Daraus entstehen medizinethisch problematische Situationen. Fraglich ist deshalb, ob das Schweizer Gesetz zur Invalidenversicherung in der heutigen Form überhaupt kompatibel ist mit internationalen Normen wie der Strassburger Konvention für Menschenrechte.

\section{Die soziologische und die politische Dimension}

Wenn sich die Bürgerinnen und Bürger nicht mehr gewiss sein können, dass im Notfall für sie gesorgt wird, so schadet das dem Zusammenhalt und stört den sozialen Frieden. Die Politik erwartet von den Behörden, ihre unabhängige Position bei der Einschätzung der Rentenberechtigung aufzugeben, damit Rentenentzug effektiver durchgeführt werden kann. Dies mit Erfolg, wie die letzten Jahre gezeigt haben, jedoch mit enormen Kollateralschäden. Die Behörden setzen alles daran, eine Person des Betrugs zu überführen, und 
wenden dabei auch ungeniert forensische Verfahren an, insbesondere Verstrickung der Personen in Widersprüche, verdeckte Ermittlungen und verdeckte medizinische Testverfahren (Medikamentenspiegel). Die weitere Konzertierung des Vorgehens umfasst die Infragestellung der Expertise der behandelnden Kollegen, den Einkauf von Gutachten zu eindeutig zu hohen Preisen, das «down-coding» von Beschwerden mittels verschiedener Tests oder mittels haltloser Anschuldigungen wie Simulation oder Aggravation. Ziel dieser

\section{Das System der IV-Renten existiert zwar, diejenigen, die die Renten benötigen, erhalten sie aber nicht oder nicht mehr.}

Aktivitäten ist die systematische Zerstörung des Rentenanspruchs anhand der Umkehr von Tatsachen. Resultat: Das System der IV-Renten existiert zwar, diejenigen, die die Renten benötigen, erhalten sie aber nicht oder nicht mehr. Damit werden die Kosten auf andere Stellen verlagert, die Gemeinden und die Spitäler. Im Hinblick auf das Referendum gegen die neue Erweiterung des ATSG-Gesetzes ist es angebracht, dass die Desinformation zu Scheininvaliden in der Bevölkerung bekannt wird, denn schliesslich sind auch die Bürgerinnen und Bürger diejenigen, welche die Zusatzkosten bezahlen, die durch politische und behördliche Fehler entstehen.

\section{Vorschlag Gesetzesänderung}

Betrügende IV-Rentenbezüger sind selten (0,3\%). Die fehlende Transparenz bei der Entscheidung zur Anordnung von Überprüfungen jeglicher Art, insbesondere auch von Observationen, obliegt heute der Versicherung/Gemeinde, welche in diesem Kontext nicht als «unabhängige» Behörde agiert und einen Interessenkonflikt hat. Dies widerspricht rechtsstaatlichen Grundprinzipien. Die heutige Praxis bei den Überprüfungen sowie die Anpassungen des Gesetzes zu den
Observationen (ATSG Art. 43ff.) dienen nicht dazu, Betrüger zu finden, sondern möglichst viele Renten zu kürzen, die eigentlich zu Recht gesprochen wurden. Dieser Rückschluss wird unterstützt durch das Vorgehen der IV, weder eine wissenschaftliche Begleitung ihrer Methoden und Prozesse zu betreiben, noch eine objektive Messung der Folgen und der Schäden zuzulassen. Ein unhaltbarer Zustand in einer aufgeklärten Wissensgesellschaft, gestützt durch eine mangelhafte Gesetzesgrundlage. Wir schlagen deshalb eine Änderung von Art. 43b vor. Diese Anpassung, wonach nur eine unabhängige paritätische Kommission eine Überprüfung anordnen darf, dient dazu, den Missbrauch von Überprüfungen zu verhindern und die Gründe für die Anordnungen transparent zu gestalten sowie eine Begleitforschung zur Häufigkeit von tatsächlichem Missbrauch auf nationaler Ebene einzuleiten. Unser Vorschlag für eine entsprechende Gesetzesänderung ATSG Art. 43 Abs. 2: "Für die Anordnung der Observation ist eine von den Versicherern teils unabhängige paritätische Kommission zuständig. Diese setzt sich zusammen aus einem Vertreter der FMH, einem Vertreter der Versicherer und einem Vertreter des Angeschuldigten nach vorheriger Anhörung des Angeschuldigten, sofern die Kommission nicht auch ohne Anhörung auf eine Observation verzichten will. Bei Uneinigkeit darf höchstens ein Vertreter der Kommission überstimmt werden.»

\section{Fazit}

Zusammenfassend ist festzuhalten, dass sich aus unserer Sicht die Institution der Invalidenversicherung in eine medizinethisch unhaltbare Richtung bewegt, indem hilfsbedürftigen Bürgerinnen und Bürgern nicht nur die ihnen zustehende Hilfe verweigert, sondern auch der Gesundheitszustand dieser Patienten durch behördliche Massnahmen verschlechtert wird.

\section{Bildnachweis}

Eigene Darstellung 